\title{
Electrically tunable diffuser for holographic multiplexing storage
}

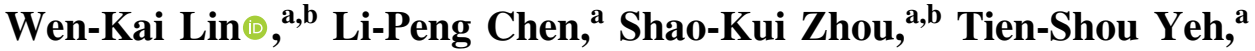 \\ and Wei-Chia $\mathrm{Su}^{\mathrm{a}, *}$ \\ ${ }^{a}$ National Changhua University of Education, Graduate Institute of Photonics, \\ Changhua, Taiwan \\ ${ }^{b}$ National Yang Ming Chiao Tung University, College of Photonics, Tainan, Taiwan
}

\begin{abstract}
A polymer-dispersed liquid crystal (PDLC) device is fabricated to serve as a diffuser for random phase encoding of reference beams in holographic storage system. By tuning the applied voltage on the PDLC device, three individual random phase reference waves which are orthogonal to each other can be generated. Therefore, holographic multiplexing scheme can be performed using voltage modulation on the PDLC diffuser. In addition, shift multiplexing technique still can be performed in the storage system. When compared with conventional shift multiplexing, using electrically tunable PDLC diffuser offers additional multiplexing mechanism in the presented technique. Using the proposed PDLC diffuser, the multiplexing numbers can be enhanced three times owing to voltage modulation. A holographic storage system implemented using shift multiplexing and voltage modulation on PDLC diffuser is demonstrated. (C) The Authors. Published by SPIE under a Creative Commons Attribution 4.0 Unported License. Distribution or reproduction of this work in whole or in part requires full attribution of the original publication, including its DOI. [DOI: 10.1117/1.OE.60.7.075105]
\end{abstract}

Keywords: diffuser; liquid crystals; holography; holographic storage; random phase encoding; random phase-shifting multiplexing; speckle-shift multiplexing.

Paper 20210457 received Apr. 28, 2021; accepted for publication Jul. 9, 2021; published online Jul. 26, 2021.

\section{Introduction}

Volume holographic storage (VHS) is considered as a potential candidate for optical data storage in the next generation owing to its high storage capacity and fast access rate. ${ }^{1-3}$ In recent years, several materials that can apply to VHS, such as dye-doped acrylic, photopolymer, polymer-doped liquid crystal (LC), and photorefractive crystal, have been researched well. ${ }^{4-10}$ Achievement of high storage capacity of VHS relies on the implementation of multiplexing techniques, such as wavelength multiplexing, angle multiplexing, shift multiplexing, and phase-code multiplexing, ${ }^{9-18}$ which enable storage of multiple images in the same area or volume becomes possible. Among these techniques, phase-code multiplexing is an interesting technique because it offers multiplexing storage mechanism for thin holograms without Bragg selectivity.

Multiplexing mechanism of phase coding in holographic storage is based on the property that reference waves are orthogonal to each other and therefore the crosstalk between sequent recordings is suppressed. Typically, there are two kinds of phase-code multiplexing method. One is orthogonal phase multiplexing and the other is random phase multiplexing. For orthogonal phase code multiplexing, phase encoding can be implemented with a phase spatial light modulator coded with orthogonal Walsh-Hadamard functions. ${ }^{14,15}$ For random phase-code multiplexing, a sequent of random phase encoding can be performed by shifting a diffuser device such as a ground glass. The newly generated phase code is orthogonal to the previous one and can be used as the reference wave to store the next new data. The orthogonal property of generated phase codes in this way has been proved and multiplexing storage based on the technique has been demonstrated ${ }^{16,17}$ In addition, random phase multiplexing also has an attractive characteristic for encryption and security. ${ }^{18-21}$ Random phase encoding technique also can be adopted to shift

*Address all correspondence to Wei-Chia Su, wcsu@cc.ncue.edu.tw 
multiplexing for holographic storage. ${ }^{19,22-25}$ In conventional random phase-shifting multiplexing system, the orthogonal reference wave can be generated via shifting tiny relative displacements between the diffuser and holographic material. Therefore, the system can record numerous images without crosstalk noise by shifting the holographic material.

In our presented technique, a polymer-dispersed liquid crystal (PDLC) device is used to serve as the required diffuser for random phase-shifting multiplexing. The transmitted random-phaseencoded wavefront is used as the reference beam for holographic storage in this system. The random phase distribution can be modified with the applied voltage on the PDLC diffuser. With proper arrangement, once the applied voltage is tuned, the newly generated random-phaseencoded wavefront becomes orthogonal to the previous one and it is not able to retrieve the previous stored hologram in the material. With this property, at least three individual random phase reference waves that are orthogonal to each other can be generated. And accordingly, three different page images can be multiplexed recorded in the same location by tuning the applied voltage on PDLC diffuser without shifting the holographic material. Simultaneously, the voltage modulation will not affect the original random phase-shifting multiplexing storage in the system.

Accordingly, a holographic storage system is successfully implemented with the combination of voltage modulation on PDLC device and random phase-shift multiplexing storage of the holographic film. The major gist of this paper is to illustrate the PDLC diffuser's advantage on multiplexing mechanism in holographic storage systems. When comparing with the tradition random phase-shifting multiplexing, the multiplexing numbers offered by proposed technique are enhanced three times owing to voltage modulation. In this paper, 12 data pages were stored within the holographic material at four recording stacks with random phase-shift multiplexing. And each stack contained three individual images recorded by voltage modulation on PDLC diffuser.

\section{PDLC Diffuser}

PDLC devices have been widely applied in electro-optical devices and displays. ${ }^{26-29}$ PDLCs usually contain approximately equal amounts of LC and polymer. As shown in Fig. 1, a cured PDLC cell consists of LC droplets embedded in a polymer matrix. The refraction index of the polymer matrix is approximately equal to the ordinary refraction index of LCs. Without applied voltage, the orientation of LCs for each droplet is randomly distributed, and the refraction index between LCs and polymer matrix is mismatched. In this state, the PDLC cell looks hazy because the incident light is scattered, and the diffuser effect is generated. In contrast, when a strong electric field is applied, the orientation of LCs in the droplets becomes parallel to the direction of the electric field if a positively anisotropic LC is employed. The PDLC cell becomes

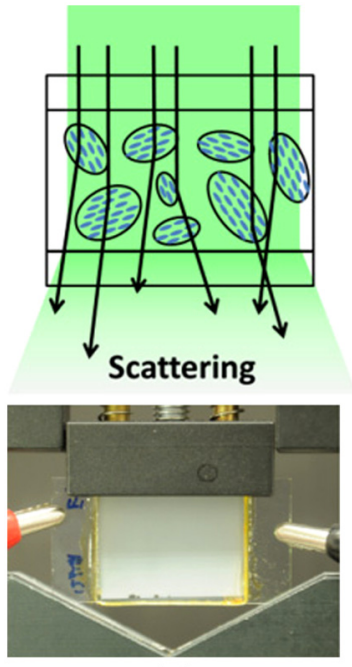

(a)

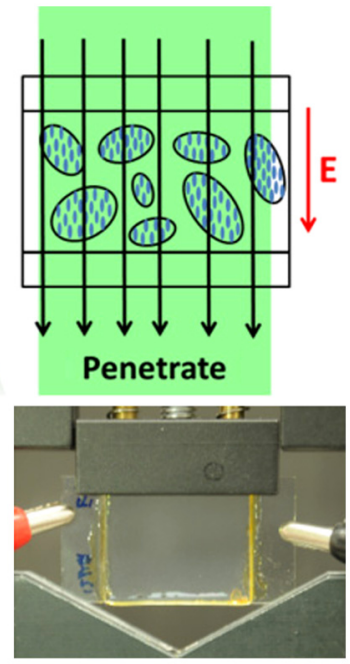

(b)

Fig. 1 (a) PDLC cell without applied voltage. (b) PDLC cell with a strong applied electric field. 


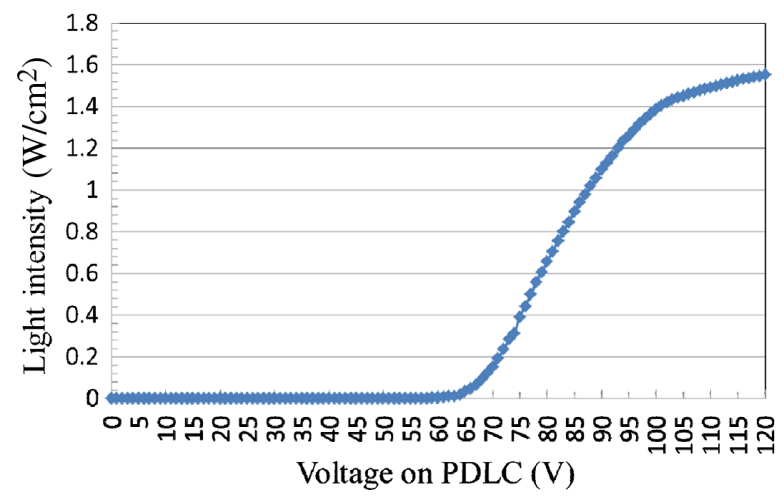

Fig. 2 The transmittance versus applied voltage curve of the PDLC device.

transparent for the incident light because the ordinary refraction index of LCs is approximately equal to that of the polymer matrix.

In this paper, PDLC diffuser is fabricated by the mixture of LC E7 (Merck) and prepolymer NOA65 (Norland) with equal amount. The mixture is injected into an empty cell fabricated with ITO glasses. After curing with UV light, the PDLC diffuser is generated. The thickness of the PDLC cell is $50 \mu \mathrm{m}$ and its dimension is $2 \mathrm{~cm} \times 3 \mathrm{~cm}$.

Figure 2 shows the transmittance versus applied voltage curves of the PDLC diffuser. The transmittance experiment of the PDLC diffuser is measured using a normal incident collimated laser beam with wavelength of $532 \mathrm{~nm}$. The measurement system is shown in Fig. 3 . The diameter of incident collimated beam is about $1 \mathrm{~cm}$. The transmission light passing through the diffuser will diverge angularly, and the distribution area of the transmission wavefront will be larger than that of power detector. A power detector with receiving area of $1 \mathrm{~cm}^{2}$ is located behind the PDLC diffuser to collect the transmission power. The input electric field is a square wave AC voltage with fixed frequency of $100 \mathrm{~Hz}$.

From Fig. 2, we can find the transmittance basically increases with the applied voltage. When the applied voltage is $<65 \mathrm{~V}$, the transmittance still approaches zero. The cell transmittance monotonically increases when the applied voltage is from 65 to $120 \mathrm{~V}$. Although the transmittance is very low and seems to keep the same when the applied voltage is $<65 \mathrm{~V}$, the phase of transmission light is indeed modulated for each different applied voltages. Furthermore, within this range, the refraction index between LCs and polymer matrix is still mismatched, and phase difference between LCs and polymer matrix is still randomly distributed in the range from 0 to $2 \pi$ for each applied voltage. Our purpose is to find at least two individual random phase reference waves which are orthogonal to each other within the applied voltage range from 0 to $65 \mathrm{~V}$.

When the applied voltage is larger than $65 \mathrm{~V}$, in the monotonically increase range, phase difference between any LCs and polymer matrix becomes smaller than $2 \pi$. The generated random phase reference wave may lack enough orthogonal property with the other one and will induce crosstalk noise for multiplexing storage. And with the increased voltage, eventual there is no phase difference between LCs and polymer matrix in the saturation state. In the saturation range, the device does not generate any effect of random phase encoding.

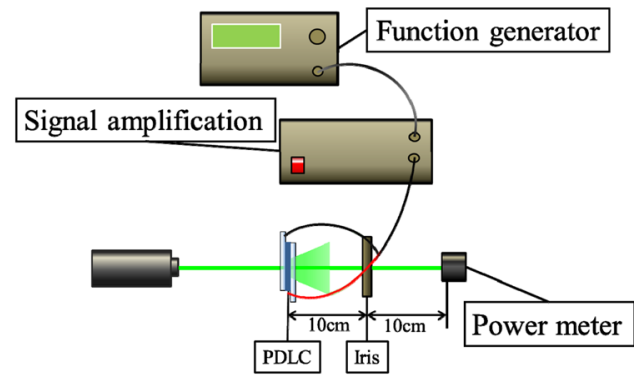

Fig. 3 The transmittance measurement system. 


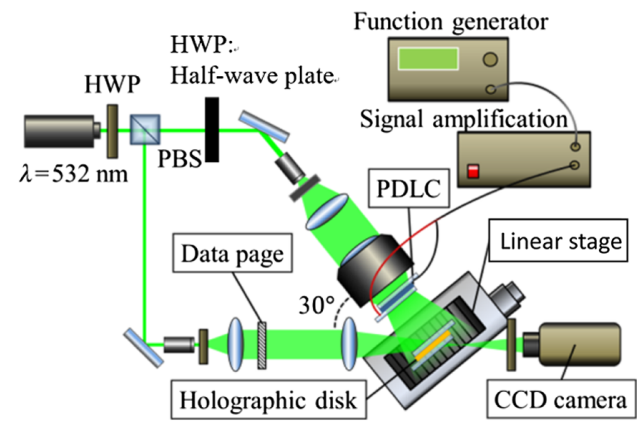

Fig. 4 The holographic multiplexing system.

\section{Holographic Multiplexing}

The holographic experiment system is shown in Fig. 4. A diode-pumped-solid-state laser with wavelength of $532 \mathrm{~nm}$ was used as the light source. The laser beam was split by beam splitter and both of them were expanded to become two collimation plane waves. Polarization states of these two waves were both s-polarized. In the arm of signal beam, an image pattern, an imaging lens, and a holographic recording material were located on the propagation path of signal beam sequentially. The incident data image was imaged on the CCD sensor. In the arm of reference beam, the collimation wave was beam-shaped to become a convergent spherical wave with a positive lens. And then the convergent spherical wave was incident on the PDLC diffuser. The illumination area of the convergent spherical wave on the PDLC diffuser was a circular shape with diameter of $15 \mathrm{~mm}$. Without the diffuser, the focus spot is located behind the diffuser with distance of $2 \mathrm{~cm}$. The transmitted wave was then used as the random-phase-encoded reference wave for holographic recording. The recording angle for the signal waves was about $30 \mathrm{deg}$ in this system. The distance between PDLC and the holographic film is $5 \mathrm{~cm}$.

The holographic film was set on a linear stage which was perpendicular to the reference wave. The holographic film is also one kind of polymer-contained material which was composed by 3 -acryloxypropyltrimethoxysilane (50.3 wt. \%), trimethylolpropane triacrylate (21.6 wt. \%), photoinitiator (3 wt. \%), and photosensitizer (0.1 wt. \%) with LC E7 (25 wt. \%) sealed in a glass cell with thickness of $20 \mu \mathrm{m}$.

\subsection{Multiplexing Storage Based on Voltage Modulation of PDLC}

In the writing process, we recorded the first image without applying any voltage on PDLC diffuser using the system as shown in Fig. 4. After recording, an electric field was applied on the PDLC device. We increased the voltage of the applied electric field and observed the diffraction of the first recorded image. We found the diffraction image will start to degrade with the increased applied voltage, and it became invisible when the applied voltage was increased to $42 \mathrm{~V}$. And then we recorded the second image using the reference wave with an applied field at $42 \mathrm{~V}$. When the recording of the second image was finished, we continued to increase the applied voltage on the PDLC diffuser and we found the diffraction of the second image degraded to vanish with an applied field of $52 \mathrm{~V}$. Then, the third image was recorded with the applied field of $52 \mathrm{~V}$. Accordingly, the applied electric field for multiplexing storage performed by voltage modulation on PDLC diffuser is selected at 0,42 , and $52 \mathrm{~V}$ for the following experiments.

\subsection{Multiplexing Storage Based on Shifting of Holographic Film}

After the recording process described in the above section, the three images' data have been stored in the same recording stack of the holographic film. The holographic film was shifted laterally by distance of $5 \mu \mathrm{m}$ using a linear stage to move to the next recording stack. The voltage modulation process for the recording of the three images in the first stack was repeated to implement storage of another three images in the second recording stack. We repeated the recording process to finish the recording in the third and fourth stack. The holographic film was shifted 


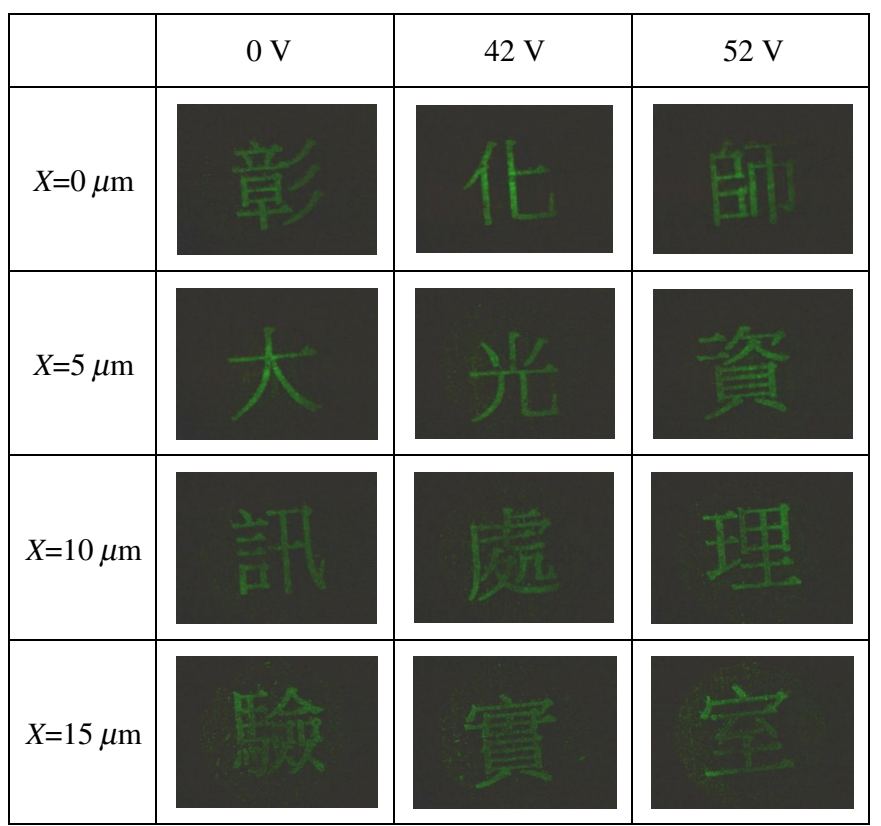

Fig. 5 The retrieved diffraction images from the holographic disk.

$5 \mu \mathrm{m}$ on each occasion before shifting to the next recording stack. As a result, each stack on the holographic film contains three individual recorded images, and their corresponding applied voltage on the PDLC device is 0,42 , and $52 \mathrm{~V}$, respectively.

\subsection{Retrieved Diffraction Images}

In the reading process, the stored 12 data images were retrieved sequentially as shown in Fig. 5 . When holographic film is shifted back to the position of corresponding stack, we can observe the diffraction of the three recorded images in turn when the applied voltage on PDLC is 0,42 , and $52 \mathrm{~V}$, respectively. From the reconstruction images, we can find there were no obvious crosstalk noises for any two adjacent voltage modulation states.

\section{Discussions}

\subsection{Shifting Selectivity}

In this section, we compare the shifting selectivity of reference waves with applied voltage at 0 , 42, and $50 \mathrm{~V}$, respectively. In the measurements, whole system setup is similar with that in Fig. 4 except the signal beam is a modified to become a collimated wave. The relationship of diffraction power and displacement of holographic film is shown in Fig. 6. From our experimental measurement results, the horizontal shifting selectivity for these three reference waves is $<3.6 \mu \mathrm{m}$. Nevertheless, to keep a higher signal-to-noise ration of diffraction image and avoid possible crosstalk noises, the shift distance for next adjacent stack in this practical demonstration system was set as $5 \mu \mathrm{m}$.

\subsection{Orthogonal Property and Crosstalk Noises}

To ensure the images recorded at 0,42 , and $52 \mathrm{~V}$ would not generate crosstalk noises with each other, we measured the power of diffraction signal at different readout conditions. Each hologram recorded at 0,42 , and $52 \mathrm{~V}$ (without voltage multiplexing storage) was individually probed by a reading wave with different applied voltage on the PDLC diffuser. The results are shown in Fig. 7. In Fig. 7(a), the diffraction signal of the hologram recorded at $0 \mathrm{~V}$ of course appears at maximum diffraction efficiency when the reading wave has applied voltage of $0 \mathrm{~V}$. But the 


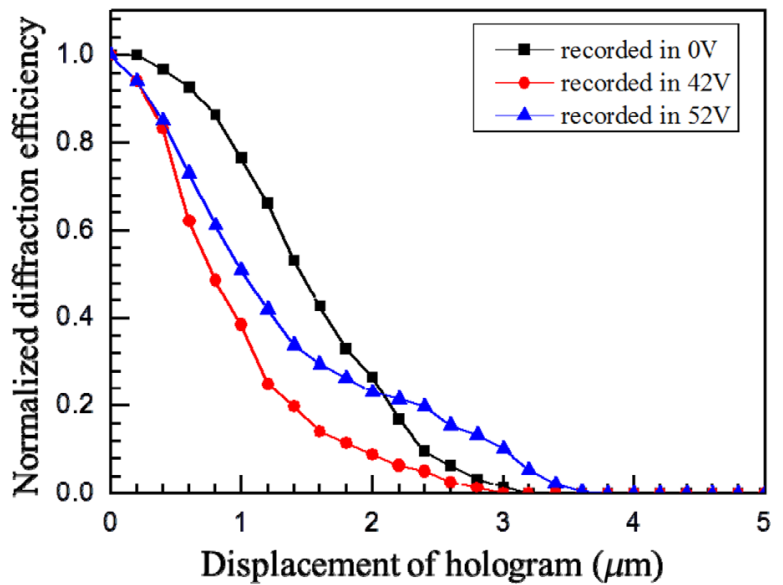

Fig. 6 Experimental results of lateral shifting selectivity for the proposed multiplexing storage system.

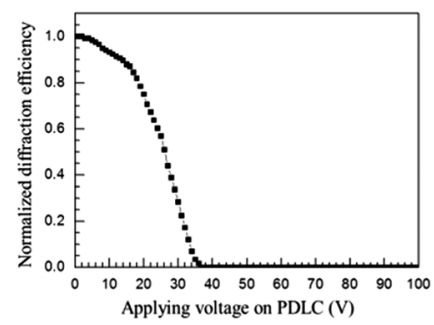

(a)

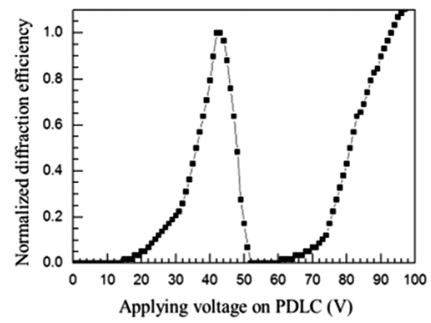

(b)

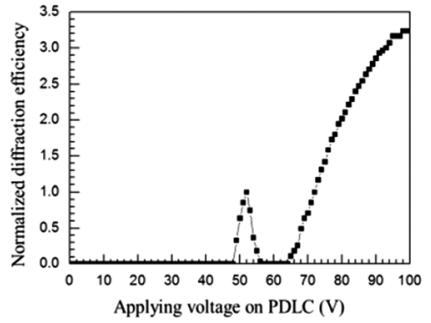

(c)

Fig. 7 The diffraction efficiency of recorded hologram probed by a reading beam with different applied voltages on PDLC device.

diffraction decreases with the increased applied voltage on the PDLC, and when the applied voltage is around $36 \mathrm{~V}$, the diffraction power approaches zero. The result seems that the voltage modulation with $36 \mathrm{~V}$ is enough to generate a new orthogonal phase wave. However, in our practical visual observation, the diffraction image will completely disappear when the applied voltage is $42 \mathrm{~V}$. In Fig. 7(b), the diffraction signal of hologram recorded at $42 \mathrm{~V}$ has a strong diffraction when the reading wave with applied voltage of $42 \mathrm{~V}$. Its diffraction disappears when the applied voltage is $0 \mathrm{~V}$. The results show these two random-phase reference waves with applied field of 0 and $42 \mathrm{~V}$ are orthogonal to each other, and therefore holograms recorded at these two states will not generate crosstalk noises. Nevertheless, when applied field is larger than $60 \mathrm{~V}$, diffraction power can be observed and measured. It means reference waves with an applied field larger than $60 \mathrm{~V}$ will induce self-crosstalk noises for the hologram recorded at $42 \mathrm{~V}$. We estimate when the applied field reaches $42 \mathrm{~V}$, the composition of the transmitted plane wave actually already contains partial plane wave. When the applied field is larger than $60 \mathrm{~V}$, the proportion of plane wave in the newly generated reference wave will increase, and therefore it induces self-crosstalk noises for the hologram recorded at $42 \mathrm{~V}$.

In Fig. 7(c), we can find the diffraction signal of hologram recorded at $52 \mathrm{~V}$ will not generate crosstalk noises for the hologram recorded at 0 and $42 \mathrm{~V}$, respectively. However, when the applied field is larger than $60 \mathrm{~V}$, self-crosstalk noises arise again for the hologram recorded at $52 \mathrm{~V}$. Noticeably, when the applied field is larger than $74 \mathrm{~V}$, the diffraction power of selfcrosstalk noise will be larger than the signal recorded at $52 \mathrm{~V}$. The reason is that the induced selfcrosstalk noises will be amplified when the hologram is probed with the strong applied electric field from the PDLC. In this condition, the reference beam has almost become a transmitted plane wave, and its power becomes much larger than the transmitted random-phase-encoded reference wave, which spreads divergently. And accordingly the induced self-crosstalk noises will be amplified and becomes larger than the signal. 
Accordingly, we conclude that there are three random phase reference waves that are orthogonal to each other can be generated in our device. With the applied voltages of 0,42 , and $52 \mathrm{~V}$ on the PDLC diffuser, the generated random phase reference waves can be used for multiplexing storage. When the applied field is larger than $60 \mathrm{~V}$, the newly generated reference wave will lack enough orthogonal property with the reference wave with applied voltages of 42 and $52 \mathrm{~V}$.

\section{Conclusion}

Based on the random phase waves generated by a PDLC diffuser and its electrically tunable property, a PDLC device is used successfully to implement a holographic multiplexing system. The presented multiplexing scheme is operated with combination of voltage modulation on PDLC device and random phase-shift multiplexing technique. Based on the random phase-shifting multiplexing system, we illustrate the voltage modulation method that can record additional images without any displacement of the holographic material. By tuning the applied voltage on the PDLC device, three individual random phase reference waves that are orthogonal to each other can be generated. Based on the mechanism, three different page images can be stored using voltage modulation on the PDLC device. The additional multiplexing ability is not able to be found from a conventional diffuser such as ground glass owing to the lack of an electrically tunable property. In the demonstrated system, 12 data pages were stored within the holographic material at four recording stacks with shift multiplexing, and three individual images were stored in each individual stack with voltage modulation on the PDLC diffuser.

\section{Acknowledgments}

This work was supported by the Ministry of Science and Technology of Taiwan under Contract No. MOST 108-2221-E-018-018-MY3. The authors declare no conflicts of interest.

\section{References}

1. G. W. Burr et al., "Volume holographic data-storage at areal density of 250-gigapixels/in. 2," Opt. Lett. 26, 444-446 (2001).

2. K. Anderson and K. Curtis, "Polytopic multiplexing," Opt. Lett. 29, 1402-1404 (2004).

3. S. S. Orlov et al., "High-transfer-rate high-capacity holographic diskdata-storage system," Appl. Opt. 43, 4902-4914 (2004).

4. V. P. Pham et al., "Real-time dynamic polarization holographic recording on auto-erasable azo-dye doped PMMA storage media," Opt. Mater. 4, 467-475 (1995).

5. V. P. Pham et al., "Novel azo dye-doped poly (methyl methacrylate) films as optical data storage media," Jpn. J. Appl. Phys. 36, 429-438 (1997).

6. I. G. Naydenova et al., "Holographic recording in nanoparticle-doped photopolymer," Proc. SPIE 6252, 625206 (2006).

7. M. S. Mahmud et al., "Holographic recording in acrylamide photopolymers: thickness limitations," Appl. Opt. 48, 2642-2648 (2009).

8. M.-S. Weiser et al., "Self-processing, diffusion-based photopolymers for holographic applications," Macromol. Symp. 296, 133-137 (2010).

9. S. Massenot et al., "Multiplexed holographic transmission gratings recorded in holographic polymer-dispersed liquid crystals: static and dynamic studies," Appl. Opt. 44, 5273-5280 (2005).

10. F. T. S. Yu et al., "Wavelength multiplexed reflection matched spatial filters using $\mathrm{LiNbO}_{3}$," Opt. Commun. 81, 343-347 (1991).

11. W. C. Su and C. M. Chen, "Angular selectivity in volume holograms with spherical reference waves implemented by rotating a mirror," Opt. Eng. 46, 015802 (2007).

12. G. J. Steckman, A. Pu, and D. Psaltis, "Storage density of shift-multiplexed holographic memory," Appl. Opt. 40, 3387-3394 (2001). 
13. C. C. Sun and W. C. Su, "Three-dimensional shifting selectivity of random phase encoding in volume holograms," Appl. Opt. 40, 1253-1260 (2001).

14. C. Denz, G. Pauliat, and G. Roosen, "Volume hologram multiplexing using a deterministic phase encoding method," Opt. Commun. 85, 171-176 (1991).

15. C. Alves, G. Pauliat, and G. Roosen, "Dynamic phase-encoding storage of 64 images in a $\mathrm{BaTiO}_{3}$ photorefractive crystal," Opt. Lett. 19, 1894-1896 (1994).

16. C. C. Sun et al., "Random phase-coded multiplexing in $\mathrm{LiNbO}_{3}$ for volume hologram storage by using a ground-glass," Opt. Quantum Electron. 28, 1551-1561 (1996).

17. J. T. LaMacchia and D. L. White, "Coded multiple exposure holograms," Appl. Opt. 7, 91-94 (1968).

18. J. F. Heanue, M. C. Bashaw, and L. Hesselink, "Encrypted holographic data storage based on orthogonal-phase-code multiplexing," Appl. Opt. 34, 6012-6015 (1995).

19. W. C. Su et al., "Security optical data storage in Fourier holograms," Appl. Opt. 51, 1297-1303 (2012).

20. W. C. Su, C. C. Sun, and W. C. Su, "Selective-encrypted holographic storage in $\mathrm{LiNbO}_{3}$ with angular multiplexing," Microwave Opt. Technol. Lett. 42, 227-230 (2004).

21. C. Denz et al., "Digital volume holographic data storage using phase-coded multiplexing," Proc. SPIE 3802, 142-147 (1999).

22. T. Nobukawa, Y. Wani, and T. Nomura, "Multiplexed recording with uncorrelated computer-generated reference patterns in coaxial holographic data storage," Opt. Lett. 40, 2161-2164 (2015).

23. T. Nishizaki, O. Matoba, and K. Nitta, "Available number of multiplexed holograms based on signal-to-noise ratio analysis in reflection-type holographic memory using threedimensional speckle-shift multiplexing," Appl. Opt. 53, 5733-5739 (2014).

24. T. Shimura et al., "Analysis of a collinear holographic storage system: introduction of pixel spread function," Opt. Lett. 31, 1208-1210 (2006).

25. X. H. Lee et al., "Random phase encoding in holographic optical storage with energyeffective phase modulation by a phase plate of micro-lens array," Opt. Commun. 287, 40-44 (2013).

26. O. Matoba et al., "Three-dimensional/two-dimensional convertible display based on computer-generated holograms and an amplitude-modulated spatial light modulator," Opt. Eng. 57, 061614 (2018).

27. M. Jamil et al., "Nanoparticle-doped polymer-dispersed liquid crystal display," Curr. Sci. 101, 1544-1552 (2011).

28. H. L. Zhang et al., "Integral imaging-based 2D/3D convertible display system by using holographic optical element and polymer dispersed liquid crystal," Opt. Lett. 44, 387-390 (2019).

29. C. T. Hsieh et al., "Polarization-independent distortion corrector fabricated using polymerdispersed liquid crystals," Appl. Opt. 53(3), 383-387 (2014).

Wen-Kai Lin received his BS degree from the Department of Applied Physics, National Pingtung University, Taiwan, in 2013, and his MS degree from the Department of Physics, National Changhua University of Education, Taiwan, in 2015. Currently, he is a PhD student at the College of Photonics, National Yang Ming Chiao Tung University, Taiwan. His interests are in optical holography, computer-generated holograms, and optical design.

Biographies of the other authors are not available. 